\title{
How Does the Internal Structure of Cardiac Muscle Cells Regulate Cellular Metabolism?
}

\author{
Shouryadipta Ghosh ${ }^{1,2}$, Eric Hanssen ${ }^{3}$, Edmund J. Crampin ${ }^{2}$ and Vijay Rajagopal ${ }^{*}$ \\ 1. Cell Structure and Mechanobiology Group, Dept. of Biomedical Engineering, Melbourne School of \\ Engineering, University of Melbourne, Melbourne, Australia. \\ 2. Systems Biology Laboratory, School of Mathematics and Statistics, and Melbourne School of \\ Engineering, University of Melbourne, Melbourne, Australia. \\ 3. Advanced Microscopy Facility, Bio21 Molecular Science and Biotechnology Institute, The University \\ of Melbourne, Melbourne, Australia. \\ * Corresponding author: vijay.rajagopal@unimelb.edu.au
}

Cardiomyocytes consist of multiple columns of mitochondria and myofibrils that run parallel to each other across the entire length of the cells. Previous studies based on electron microscopy (EM) images have revealed that this columnar mitochondrial organization can be significantly altered in diseases like type I diabetes [1]. Mitochondria in cardiomyocytes affected by diabetes form large clusters and are distributed non-uniformly across the cell. The aim of this study is to determine how these alterations affect bioenergetics and contractile behavior of cardiomyocytes. A previously published finite element (FE) model of cardiac bioenergetics, incorporating cross sectional EM images of cardiomyocytes, was used to find answers to this question [2].

Tissue blocks were prepared from the left ventricular wall of 2 control and 2 STZ induced type I diabetic Sprague Dawley rats. These blocks were imaged using two different EM techniques -2 nanometerresolution TEM (transverse electron microscopy) of individual cross sections and 50 nanometerresolution SBFSEM (serial-block-face scanning electron microscopy) of complete or partial cell volumes. A detailed description of the animal and imaging protocols are available in our previous publications [3, 4]. The collected images were automatically segmented into mitochondrial and myofibrillar regions using an image processing algorithm for SBFSEM images [3, 4]. 9 control and 9 diabetic $2 \mathrm{D}$ cross sections were randomly sampled from these image stacks to generate 2D FE meshes consisting of mitochondrial and myofibrillar regions. An experimentally validated biophysical FE model, describing mitochondrial respiration and myofibrillar ATP consumption, was simulated over these spatially detailed FE meshes [2].

The FE simulations revealed that myofibrils in diabetic cell cross sections have larger concentration gradients of metabolites such as ATP and ADP when compared to control cross sections. Fig.1 shows the results from two representative TEM cross sections. It is evident from Fig. 1 that concentration of ATP and ADP are nearly uniform across the control cell cross section. In contrast, myofibril areas of the diabetic cell cross section have different ATP and ADP concentrations depending on the proximity of mitochondria. When all 18 cross sections were considered, the average of interquartile ranges (IQR) of ADP/ATP distribution in the diabetic cells was $\sim 20 \%$ higher than that in the control cells. These concentration gradients are a consequence of mitochondrial clustering and heterogenous arrangement of mitochondria in diabetic cells. The non-uniform concentrations of ATP and ADP also resulted in a nonuniform distribution of myofibrillar ATP hydrolysis rate across the diabetic cell cross sections (Fig.1). IQRs of ATP hydrolysis rate distributions, normalized by corresponding cell cross section sizes, were $\sim 74 \%$ higher in the diabetic cells. 
These findings suggest that heterogenous mitochondrial distribution in diabetic cells can lead to a heterogenous metabolic landscape. Since ATP hydrolysis rate in myofibrils correlates with the speed of muscle shortening, different parts of diabetic cells might contract at different rate, which can decrease the energetic efficiency of the cells and damage the cell structure.

\section{References:}

[1] J Jarosz et al., American Journal of Physiology - Cell Physiology 312 (2017), p. C190.

[2] S Ghosh et al., PLOS Computational Biology 14 (2018), p. e1006640.

[3] A Hussain et al., Journal of Structural Biology, https://doi.org/10.1016/j.jsb.2018.02.005(2018), p.

[4] A Hussain, E Hanssen and V Rajagopal, Microscopy and Microanalysis 23 (2017), p. 240.

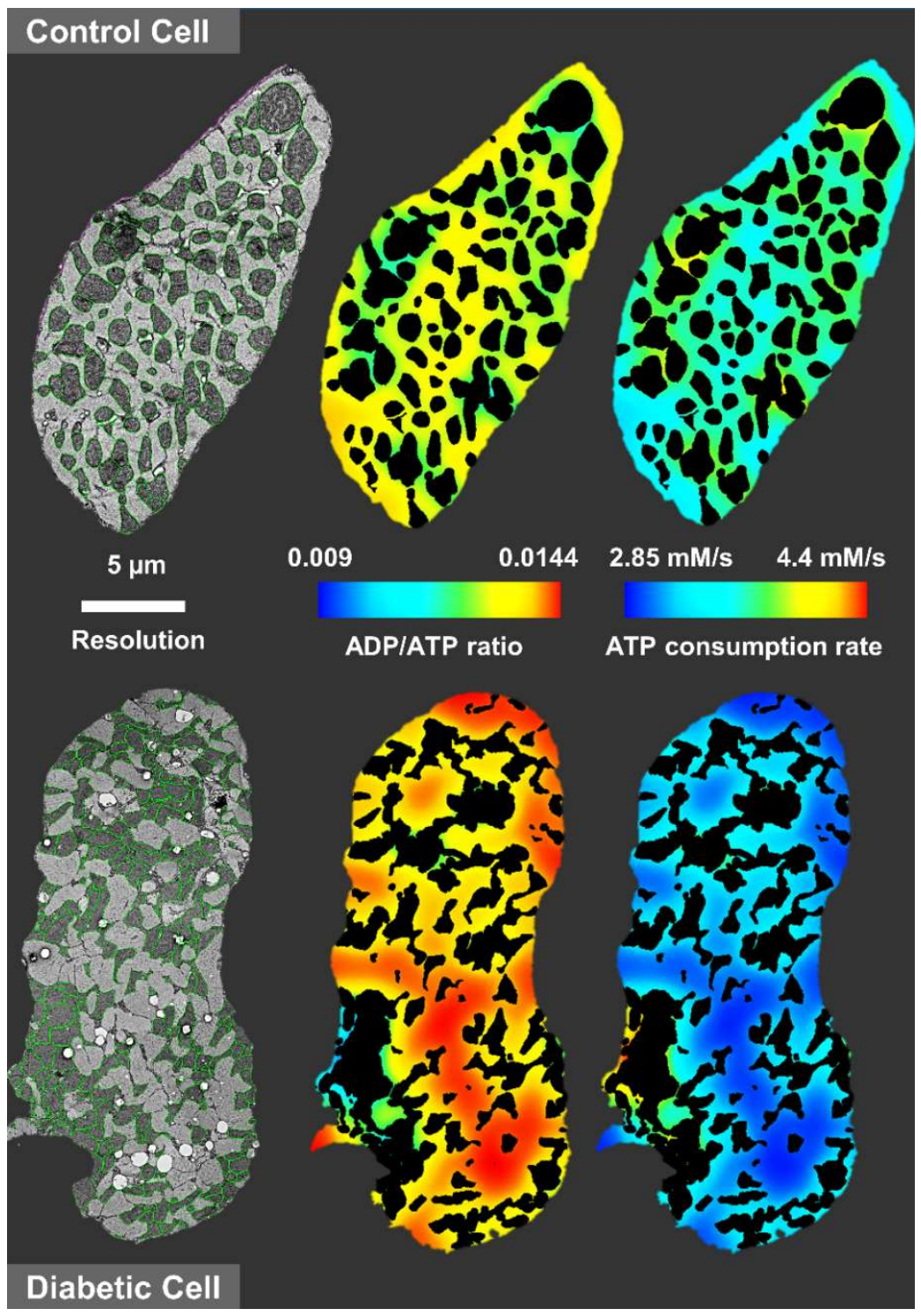

Figure 1. Arrangement of mitochondria is irregular in the type I diabetic cardiac cell. As a result, compared to the control cross section, ATP and ADP are distributed more non-uniformly in the diabetic cross section. The diabetic cross section also shows large variation in the consumption rate of ATP. 